\section{SAT0036 IMPACT OF RHEUMATOID ARTHRITIS IN LIVER DAMAGE. INVOLVEMENT OF ANTI-CITRULLINATED PROTEIN ANTIBODIES}

Iván Arias de la Rosa ${ }^{1}$, Carmen Torres-Granados ${ }^{1}$, Carlos Perez-Sanchez ${ }^{2}$, Maria del Carmen Abalos-Aguilera ${ }^{1}$, Alejandra Patiño-Trives ${ }^{1}$, Maria Luque-Tevar ${ }^{1}$, Alejandro Ibañez-Costa ${ }^{1}$, Rafaela Ortega Castro ${ }^{1}$, Jerusalem Calvo Gutierrez ${ }^{1}$, Eduardo Collantes Estevez ${ }^{1}$, Alejandro Escudero Contreras ${ }^{1}$, Chary LopezPedrera ${ }^{1}$, Nuria Barbarroja Puerto ${ }^{1}{ }^{1}$ IMIBIC/Reina Sofia Hospital/University of Cordoba, CORDOBA, Spain; ${ }^{2}$ Department of Medicine, University of Cambridge, School of Clinical Medicine, Addenbroke's Hospital, Cambridge Institute for Medical Research, CORDOBA, Spain

Background: Liver damage in rheumatoid arthritis (RA) is most common in the form of asymptomatic abnormal liver biopsies. It is difficult to differentiate between hepatic manifestations of the primary disease and potential hepatotoxicity of the therapies. Inflammation, oxidative stress, apoptosis and loss of lipid droplets are involved in the hepatic fibrogenesis

Objectives: 1)To analyze the impact of RA in the liver function and 2)To evaluate the direct effect of anti-citrullinated protein antibodies (ACPAs) in the liver fibrosis.

Methods: 150 RA patients and 100 healthy donors (HD) were included. Aspartate aminotransferase (AST), alanine aminotransferase (ALT), phosphatase alkaline (ALP), albumin and levels of autoantibodies and inflammatory markers were evaluated in serum. In vitro studies: Hep G2 cells were treated with IgG-ACPAs isolated from RA patients. Molecules involved in lipid metabolism, insulin resistance, oxidative stress and inflammation were analyzed by RT-PCR and Western blot. Activation of intracellular pathways involved in fibrogenesis were analyzed. Lipid accumulation was evaluated by fluorescence microscopy. Mouse model: 20 CB57J/BL mice were used; 5 mice were used as non-diseased group, and 15 were used in CIA modelling. Liver samples were collected. Genes involved in insulin signal, lipid accumulation, macrophage infiltration and polarization and inflammation were evaluated. Activation of intracellular pathways related to fibrogenesis were analyzed. Immunohistochemistry was used to evaluate the percentage of fibrotic cells.

Results: Within the normal range of hepatic enzymes, the percentage of RA patients with levels of AST, ALT and ALP above the mean was significantly higher compared to HD. In contrast, percentage of RA patients displaying levels of albumin bellow the mean was significantly elevated compared to HD. Differences remained significant after adjusting for potential confounders (treatment). Moreover, high levels of AST and ALT were associated with ACPAs and inflammatory markers. IgG-ACPAs induced the expression of inflammatory and oxidative stress markers and decreased genes involved in insulin signal and lipid accumulation in Hep G2 cells. In addition, lipid content decreased after IgG-ACPAs treatment. The phosphorylation of intracellular pathways involved in fibrogenesis was modulated by Ig-ACPAs. The induction of arthritis in mice elevated inflammatory cytokines and markers of macrophages presence and polarization state M1 and reduced genes related to lipid droplets in the liver. Phosphorylation of ERK and mTOR was increased in the liver of CIA mice. Additionally, the percentage of cells positive for $\alpha$-smoth muscle antibody was increased in the liver of ClA mice.

Conclusion: (1) RA patients displayed a subclinical alteration of the hepatic enzymes levels associated with levels of autoantibodies ACPAs, which may suggest that RA is associated with an abnormal liver function induced by autoantibodies. (2) ACPAs may induce alterations in hepatic cells, increasing inflammation and oxidative stress, reducing lipid accumulation and activating intracellular pathways, processes closely involved in fibrogenesis. (3) In a ClA mice, arthritis induced inflammation, infiltration of macrophages, reduction of genes involved in lipid accumulation and activation of intracellular pathways involved in fibrogenesis Acknowledgement: Funded by ISCIII (CP15/00158 and PI17/01316) cofunded with FEDER.

Disclosure of Interests: None declared

DOI: 10.1136/annrheumdis-2019-eular.5641

\section{SAT0037 INCREASED CARDIOMETABOLIC RISK FACTORS ARE RELATED TO THE ABNORMAL ADIPOCYTOKINE PROFILE AND AUTOIMMUNITY IN RHEUMATOID ARTHRITIS. MODULATION BY TNFALPHA AND IL6R INHIBITORS}

Iván Arias de la Rosa ${ }^{1}$, Maria del Carmen Abalos-Aguilera ${ }^{1}$, Rafaela Ortega Castro ${ }^{1}$, Jerusalem Calvo Gutierrez ${ }^{1}$, Carlos Perez-Sanchez ${ }^{2}$, Alejandro Ibañez-Costa ${ }^{1}$, Alejandra Patiño-Trives ${ }^{1}$, Maria Luque-Tevar ${ }^{1}$, Eduardo Collantes Estevez ${ }^{1}$, Chary Lopez-Pedrera ${ }^{1}$, Alejandro Escudero Contreras ${ }^{1}$, Nuria Barbarroja Puerto ${ }^{1} .{ }^{1}$ MIBIC/Reina Sofia Hospital/University of Cordoba, CORDOBA, Spain; ${ }^{2}$ Deparment of Medicine, University of Cambridge, School of Clinical Medicine, Addenbroke's Hospital, Cambridge Institute for Medical Research, Cambridge, UK, Cambridge, United Kingdom

Background: Rheumatoid arthritis (RA) is a systemic autoimmune disease characterized by excess morbidity and mortality from cardiovascular disease. In addition, metabolic alterations have been observed in these patients, which significantly contributes to the cardiovascular risk burden Thus, the identification of therapies able to mitigate the cardiometabolic alterations in RA is essential. In addition, numerous studies suggest that cardiometabolic risks are mediated through adipocytokines. However, this relationship is not completely defined in RA

Objectives: 1) To evaluate the relationship among cardiometabolic risk factors and the levels of adipocytokines and autoantibodies in RA patients. 2) To analyze the effects anti-TNF $\alpha$ and anti-IL6 therapies on the cardiometabolic alterations.

Methods: 1.- A cross-sectional study including 100 RA patients and 50 age-matched healthy donors was carried out. Different parameters related to the cardiometabolic risk were analyzed, including: lipid profile, atherogenic index, ratio $A p o B / A p o A$, insulin resistance (IR), obesity, hypertension, and the SCORE. Levels of adipocytokines (TNF $\alpha$, IL6, IL1 $\beta$, visfatin, adiponectin, leptin and resistin) were evaluated in serum. Carotid intima media thickness (CIMT) was evaluated as atherosclerosis marker. 2.-A prospective study in $15 \mathrm{RA}$ patients before and after 3 months of anti-TNF $\alpha$ therapy and 15 RA patients before and after 3 months of treatment with tocilizumab (TCZ) was performed. All the parameters evaluated in the cross-sectional cohort were tested in this prospective study.

Results: RA patients had elevated levels of leptin/adiponectin ratio, visfatin, resistin and inflammatory markers in serum. Our cohort of RA patients displayed increased rates of cardiometabolic risk factors, such as insulin resistance, hypertension, SCORE, pathologic CIMT, atherogenic index and ratio apoB/apoA. The alteration in the levels of adipocytokines were closely related to the autoimmunity, disease activity and clinical inflammatory markers. Of note, visfatin and C3 complement levels were determinant for IR, high levels of SCORE, increased parameters of CVD risk defined by apoB/apoA ratio and pathologic CIMT.

Both biological therapies reduced clinical inflammatory markers and disease activity after 3 months of treatment. Anti-TNF $\alpha$ therapy modulated the cytoadipokine profile, reducing serum levels of IL-6, IL-1 $1 \beta$, resistin and visfatin, decreasing IR.

After treatment with tocilizumab, serum levels of C3 complement, IL-1 $\beta$ and resistin were reduced.

Conclusion: 1) Altered adipocytokine profile is closely related to the increased cardiometabolic risk factors associated with RA, where autoimmunity and systemic inflammation play a key role. 2) AntiTNF $\alpha$ and anti-IL6R therapies, administered for 3 months, could have beneficial effects in the reduction of cardiometabolic risk factors in RA.

Acknowledgement: Funded by ISCIII (PI18/00837, CP15/00158, PI17/ 01316 and RIER RD16/0012/0015) co-funded with FEDER

Disclosure of Interests: None declared

DOI: 10.1136/annrheumdis-2019-eular.5532 